\title{
Can Formal Institutions Lead to the Spillover Effect of Cooperation?
}

\author{
Rati Mekvabishvili \\ Faculty of Economic and Business, Ivane Javakhishvili Tbilisi State University, Tbilisi, Georgia \\ Email: rati.mekvabishvili@eab.tsu.edu.ge
}

How to cite this paper: Mekvabishvili, R. (2021). Can Formal Institutions Lead to the Spillover Effect of Cooperation? Theoretical Economics Letters, 11, 186-193. https://doi.org/10.4236/tel.2021.112013

Received: February 16, 2021

Accepted: March 28, 2021

Published: March 31, 2021

Copyright (c) 2021 by author(s) and Scientific Research Publishing Inc. This work is licensed under the Creative Commons Attribution International License (CC BY 4.0).

http://creativecommons.org/licenses/by/4.0/

\begin{abstract}
Can formal institutions shape prosocial behavior and lead to the spillover effect of cooperation? To explore this question, we experimentally test the spillover-based theory in a novel context. We measured the spillover effect on cooperation in the same domain measured by the repeated anonymous public goods game. We found strong evidence of altruism. Our results are inconsistent with prediction of the spillover-based theory. Our finding suggests that exposure to strong formal institutions that provide top-down motivation for cooperation substantially improves cooperation in their presence, but do not seem to lead to more prosociality after their absence.
\end{abstract}

\section{Keywords}

Centralized Punishment, Spillover, Public Goods, Cooperation

\section{Introduction}

In economic literature, the "tragedy of the commons" is probably the best-known example of when collective welfare is jeopardized by individual self-interest (Hardin, 1968). The "free rider problem", arising from the fact that an individual may be able to obtain the benefits of a good without contributing to its cost, is discussed in a number of different contexts. Provision of public goods is vulnerable to free riding; one can receive a benefit without contributing towards the cost of its production. Thus, there exists a tension between individual and collective interest, which is typical for many cooperation problems in economics.

Society has responded to the "free rider problem" by developing formal institutions precisely to prevent this type of opportunistic behavior. Public agencies, courts, and police are all examples of formal institutions put in place by society in order to provide protection against free riding behavior. Indeed, Hobbes ar- 
gued that people benefit if there is a powerful state in place to regulate behavior, thereby enabling people to invest effort in achieving collective welfare (Hobbes, 1996 [1651]). Moreover, when cooperation and prosocial behavior prevail in society, provision of public goods is more cost-efficient for the state (Gintis et al., 2005).

Our aim is to understand the impact and consequences of formal institutions chosen by society to protect against opportunistic behavior. A useful tool to analyze this social dilemma is the linear public goods game (Ledyard, 1995). In particular, with the public goods game (PGG), we experimentally test a prediction of the spillover-based theory that implies that strong formal institutions that incentivize cooperation not only restrain free riding, but boost cooperation via spillovers (Rand et al., 2014; Stagnaro et al., 2017). Thus, people exposed to strong formal institutions that provide top-down motivation for cooperation will develop more cooperative and prosocial behavior. In other words, people tend to become more prosocial and cooperative even in the absence of strong formal institutions. Formal institutions are strong when they are not corrupt, efficiently discipline free riders, and provide public goods (Stagnaro et al., 2017).

Empirical support for the spillover-based theory comes from evidence that experimentally manipulated institutional strength in the PGG, with a centralized punishment mechanism, led to significantly more prosociality in a subsequent one-shot dictator game, compared to a no-punishment control treatment (Stagnaro et al., 2017). In the study, the emphasis was on the finding that strong formal institutions that incentivized prosociality positively affected prosociality in a novel context. Namely, the motivation to cooperate in the PGG domain increased subsequent giving in the dictator game domain.

We build our study on the experimental study of Stagnaro et al., 2017, who experimentally induced institutional strength by introducing different levels of probabilities of centralized punishment mechanisms. The higher was the probability of sanctioning a free rider, the stronger was the formal institution. Therefore, in order to induce strong formal institutions, we set the centralized punishment probability at $90 \%$. Unlike Stagnaro et al., 2017, we measure spillover of prosociality in one domain, namely by the PGG. Our experiment consists of two treatments. In treatment (CP), experimental subjects play a two-stage repeated PGG. In the first stage, subjects play the PGG with the centralized punishment probability mechanism (CPPM). In the second stage, the CPPM is removed and subjects play the same standard linear PGG. As a control treatment, we conducted a standard linear PGG of ten periods (Ledyard, 1995).

We find that a strong formal institution substantially improves cooperation. On average, in stage 1, contribution rates amount to $92 \%$ of the endowment. However, as soon as the CPPM is removed, cooperation decays. In the last period, $50 \%$ of the subjects took a free ride and only $28 \%$ cooperated fully. More importantly, in our control treatment, cooperation was significantly higher than in stage 2 of treatment (CP) (two-tailed $t$-test, $t=-7.98, p=0.000$ ). We also 
classify individuals according to a simple typology: in the control treatment, $17.5 \%$ were altruists and none were free riders; while in stage 2 of treatment (CP) without centralized punishment, altruist decreased to $7.8 \%$ and free riders increased to $6.3 \%$.

Our experimental evidence does not provide support of the spillover-based theory. In our novel context, turning from different domain settings to one domain setting, we find no evidence of spillover effects. Our results suggest that a strong formal institution does not seem to foster prosociality via spillovers in the same domain (as measured by the repeated anonymous PGG). On the other hand, the lack of a positive spillover effect could be due to insufficient duration of exposure to strong formal institutions that provide top-down motivation for cooperation. It could also be that we did not observe a spillover effect for cooperation might be due to the mechanism underlying centralized punishment probability. Further investigation of the spillover effect on cooperation in different experimental design settings is an important direction for future work.

\section{Experimental Design}

\subsection{Participants}

The experiment was conducted in Georgia via the LIONESS software platform for interactive online experiments (Arechar et al., 2018). A total of 121 subjects participated, mostly from Tbilisi State University. We prevented repeated participation by excluding duplicate IDs and IP addresses. Participants were not informed about the identity of their group members. We kept group members constant during all periods in both treatments. In total, nine experiment sessions were conducted. The control treatment with the standard public goods game (PGG) of ten periods the experiment lasted between 10 and 20 minutes and participants earned, on average, 13.7 GEL (4.2 USD at that time). In treatment (CP), since it was composed of two stages and subjects played twenty rounds in total, the experiment lasted 30 to 40 minutes and participants earned, on average, 20.7 GEL (6.3 USD).

\subsection{Method}

Our work is closely related to the study of (Stagnaro et al., 2017) (henceforth SAR). We modified the experimental design of SAR in several ways. Unlike SAR, we kept the PGG in both stages in order to measure cooperation in the same domain and to avoid potential cultural context-specific variations caused by introduction of different games (Henrich and Henrich, 2007). Next, we adjusted the experimental parameter of the endowment to 20 tokens as the established amount level in most PGG experiment literature. Next, in SAR, the centralized punishment probability mechanism (CPPM) is presented as the "inspection" of contributions, and if a player did not contribute fully to the public good, when inspected with a certain probability, a deduction of points followed. 
In our design, inspection was an act representing that the player's contribution was just observed. The penalty was levied to free riders only if they were inspected. Thus, in our setting, the penalty probability is conditioned by the inspection probability. We set both probabilities of inspection and penalty at the same high level of $90 \%$. The reason we introduced inspection and penalty separately is to bring more accurate framing to the notion of strong formal institutions. The logic is as follows: if one is assigned to protect provision of public goods and does not discipline free riders simply because the opportunistic act was not observed, it is qualitatively different from the case where the opportunistic act is detected, but not disciplined. In other words, one's trustfulness is questioned at a different level if they are aware of a violation and do not act rather than if they do not act, because they are not aware of it. An example could be a police officer that observes a violation, but does not take measures to stop it.

\subsection{Payoff Mechanism}

In treatment (CP), subjects play two-stage PGG with ten periods each. In stage 1, subjects in groups of four play a standard linear PGG with the centralized punishment mechanism. Subjects simultaneously decide how much of 20 endowment points to keep or invest into the public good in each period. Payoff is determined by $\pi=\pi_{i}^{1}-2 *\left(20-g_{i}\right) * P(A \mid B)$ where $\pi_{i}^{1}=20-g_{i}+0.375 \sum_{i=1}^{n} g_{i}$, $g_{i}$ is subject is contribution to the public good, and 0.375 is the marginal percapita return of contributing to the public good. $P(A)$ is the probability that penalty will be imposed, given the probability $P(B)$ that contribution will be inspected, where $P(A)$ and $P(B)$ both equal to 0.9 . In stage 2 , the centralized punishment mechanism is abolished, and participants play a standard PGG. In stage 2 and in the control treatment experiment, the payoff is determined by $\pi_{i}^{1}=20-g_{i}+0.375 \sum_{i=1}^{n} g_{i}$.

\subsection{Information Conditions}

In treatment $(\mathrm{CP})$, in stage 1 , in each round there was a $90 \%$ probability that each of the participants' contributions would be inspected and penalized. If inspected, participants were penalized with $90 \%$ probability if they had not contributed the maximum amount of points endowed (they lost points twice the number of points below the maximum contribution of 20 points). In both treatments, players made their contribution decisions simultaneously; once the decisions were made, they were informed about their group members' contributions. However, in treatment (CP), subjects were not informed about the inspection and penalty activities of their group members. They knew of only their own inspection and penalty activities in each period.

To ensure that participants did not have varying expectations of the length of the game, the total number of rounds was made public knowledge in both treatments. Importantly, for treatment (CP), in stage 2 the removal of the inspection and penalty mechanisms was not disclosed to the participants in advance, but 
introduced just before the start of stage 2 .

To maximize data quality, we required game comprehension prior to playing the PGG: after reading the instructions, participants could not advance to the game until they correctly answered all control questions (they were allowed an unlimited number of attempts).

\section{Predictions}

In the control treatment, the spillover-based theory predicts that contributions will converge to zero and the cooperation level should be lower than in stage 2 of treatment (CP). In stage 1 of treatment (CP), we should observe a high level of cooperation under the incentive of high inspection and penalty probability. Next, in stage 2 of treatment (CP), the cooperation level should be higher than in the control treatment, as a result of the spillover effect of strong state institutions that incentivize cooperation.

\section{Results}

Figure 1 summarizes the behavior in both treatments. In the left panel, we observe average contributions in the control treatment and in stage 1 of treatment (CP). In the right panel, we depict the mean contributions of stage 2 of treatment $(\mathrm{CP})$.

The existence of inspection and penalty probabilities causes a significant rise in the average contribution level. On average, contribution rates amount to $92 \%$ (18.5 points, standard deviation 0.66 ) of the endowment in stage 1 . In stage 1 , mean contributions start relatively high and converge to socially beneficial equilibria, although they experience (per the endgame effect) a slight drop in the last period (10). This result shows that CPPM mechanism in stage 1 of treatment (CP) induces behavior in line with the theoretical predictions. In stage 2 , as soon as the centralized punishment mechanism is removed, mean contributions decay and start to converge to Nash equilibria of zero contributions. The difference between the stages is highly significant (two-tailed paired $t$-test, $t=15.48, p=$ 0.000 ). This indicates that the removal of a centralized punishment triggers the drawing power of the equilibrium with free riding.

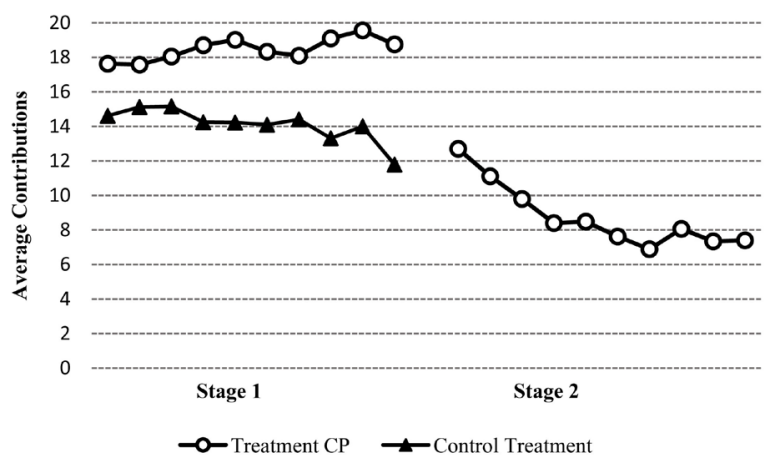

Figure 1. Average contributions over periods. 
Although the mean contributions in the control treatment overall exhibit a surprisingly flat pattern, voluntary contributions across all rounds remain quite high, well above $50 \%$. This result is not in line of theoretical prediction. Indeed, the mean contributions in the control treatment appeared highest among 16 different pool results of the cross-cultural experiment done by Herman et al., 2008 (Mekvabishvili, forthcoming). This finding provides strong evidence of altruism.

In the last round, typical for this kind of public goods experiment, there is a pronounced endgame effect with a sharp drop (two-tailed paired t-test, differences between round 1 and round 10, $t=2.06, p=0.042$ ). In the last period, 22\% percent of the subjects contributed zero. However, average contributions in the control treatment are significantly higher than in stage 2 of treatment (CP) (two-tailed $t$-test, $t=-7.98, p=0.000)$. In the control treatment, the average contribution is 14.1 points, while it is only 8.8 points in stage 2 of treatment (CP). This result is in contradiction with the prediction of spillover-based theory.

Next, we analyzed our data to track individual behavior of players, taking into account the typology of players' behavior classified in the experimental literature. We specifically look for altruist types, those who contribute the full endowment in all periods; and free riders, those who contributed zero in all periods.

Figure 2 depicts player types observed in control treatment and in stage 2 of treatment $(\mathrm{CP})$. On the horizontal axis, two treatments are displayed and on vertical axis the percentage share of altruist and free rider player types of each treatment are given. Figure 2 reveals that, in the control treatment, we observe $17.5 \%$ of altruist types and $0 \%$ of free rider types of players, while in stage 2 of treatment $(\mathrm{CP})$, the share of altruist types is only $7.8 \%$ and the share of free riders increases to $6.3 \%$.

In addition, we checked the distribution of contributions in stage 2 of treatment $(\mathrm{CP})$ and in the control treatment. We classify the contributions into four categories: 1) zero, 2) full contribution, 3) contribution equal to or more than $50 \%$ of the endowment, and 4) contribution of less than $50 \%$ of the endowment. Figure 3 depicts the distribution of decisions of all subjects across all periods over these four categories.

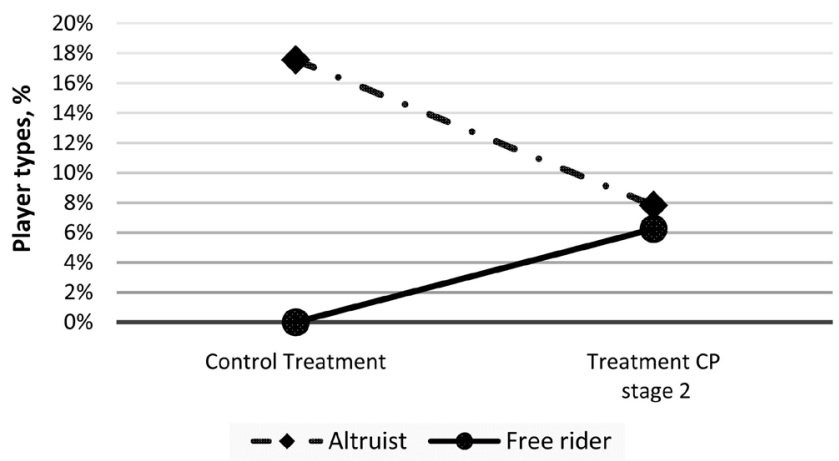

Figure 2. Player types in control treatment and in stage 2 of treatment (CP). 


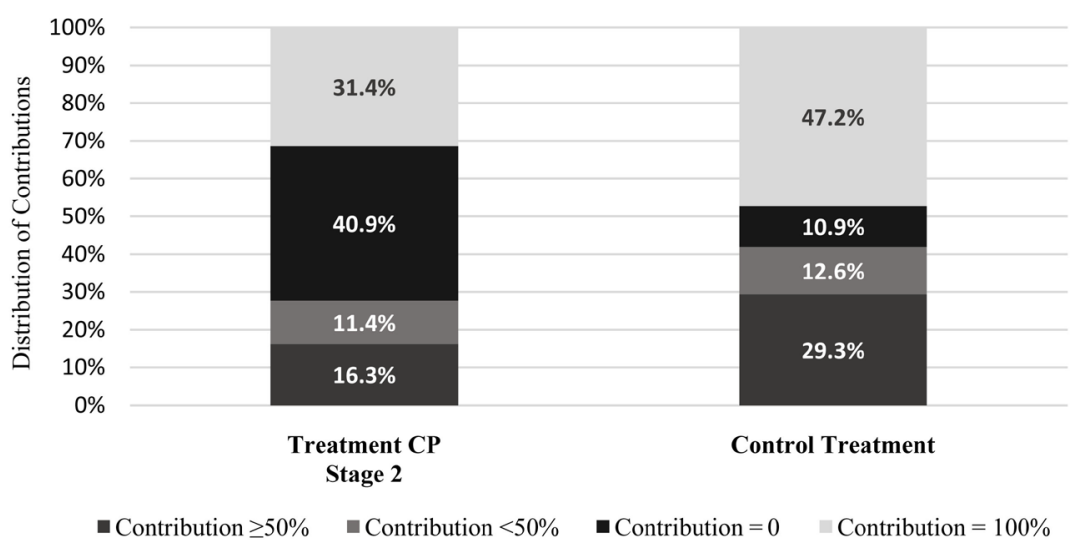

Figure 3. Distribution of total contributions in control treatment and in stage 2 of treatment $(\mathrm{CP})$.

In Figure 3, in total we observe $47.2 \%$ of full contribution decisions in the control treatment, while in stage 2 of treatment $(\mathrm{CP})$, full contribution decisions were only $31.4 \%$. Moreover, there is a dramatic increase of zero contribution decisions in stage 2 of treatment (CP), in contrast to the control treatment (from $10.9 \%$ to $40.9 \%)$.

\section{Summary and Conclusion}

This paper has shown that the centralized punishment mechanism with high probability substantially improves provision of public goods. This finding replicates the experimental evidence by Stagnaro et al., 2017. Centralized punishment with high probability constitutes a credible threat for potential free riders and enforces high cooperation, whereas the same subjects converge toward full defection when the centralized punishment mechanism is removed.

However, in our novel experimental settings, we do not observe the spillover effect on prosociality. After removal of the CPPM, the mean contributions are significantly lower than the contribution level in the control treatment. Thus, our results suggest that, under conditions of the same context and domain (i.e. PGG), exposure to strong formal institutions that provide top-down motivation for cooperation does not seem to lead to more prosociality after its absence.

Arguably, the fact that cooperation spillovers only occurred in the context of different domains, but were not observed in the same domain, could be due to insufficient exposure of the subject to top-down incentives for cooperation or the mechanism underlying the centralized punishment.

In our view, our novel finding contributes to the understanding of the role of formal institutions in protecting against opportunistic behavior and in shaping individuals' prosociality. Firstly, we provide strong evidence of altruism. Secondly, strong formal institutions can ensure high-level of cooperation. Thirdly, and not lastly, our results tentatively indicate that extrinsic motivations provided by strong state institution might undermine one's intrinsic motives to behave prosocially. 


\section{Acknowledgements}

I am indebted to Prof. Chris Starmer for his valuable comments and discussions.

This work was supported by Shota Rustaveli National Science Foundation (SRNSF), grant PHDF-19-321, "Prosocial Behavior in Economics and Influence of State Institutions: Case of Georgia”.

\section{Conflicts of Interest}

The author declares no conflicts of interest regarding the publication of this paper.

\section{References}

Arechar, A., Gächter, S., \& Molleman L. (2018). Conducting Interactive Experiments Online. Experimental Economics, 21, 99-131. https://doi.org/10.1007/s10683-017-9527-2

Gintis, H., Bowles, S., Boyd, R. T., \& Fehr, E. (2005). Moral Sentiments and Material Interests: The Foundations of Cooperation in Economic Life (Vol. 6). Cambridge, MA: MIT Press. https://doi.org/10.7551/mitpress/4771.001.0001

Hardin, G. (1968). The Tragedy of the Commons. Science, 162, 1243-1248. https://doi.org/10.1126/science.162.3859.1243

Henrich, N., \& Henrich, J. P. (2007). Culturally Evolved Social Norms Lead to Context-Specific Cooperation. In Why Humans Cooperate: A Cultural and Evolutionary Explanation (Ch. 8, pp. 157-173). Oxford: Oxford University Press.

Herrmann, B., Thoni, C., \& Gächter, S. (2008). Antisocial Punishment across Societies. Science, 319, 1362-1367. https://doi.org/10.1126/science.1153808

Hobbes, T. (1996 [1651]). Leviathan, or the Matter, Forme, and Power of a Commonwealth Ecclesiastical and Civil. Cambridge: Cambridge University Press.

Ledyard, J. O. (1995). Public Goods: A Survey of Experimental Research. In J. Kagel, \& A. E. Roth (Eds.), The Handbook of Experimental Economics (pp. 111-194). Princeton, NJ: Princeton University Press. https://doi.org/10.2307/j.ctvzsmff5.6

Mekvabishvili, R. (Forthcoming). Georgia Leads in Prosociality: Comparison to CrossCultural Economic Experiment. Economics and Business, XII, No. 3.

Rand, D. G., Peysakhovich, A., Kraft-Todd, G., Newman, G., Wurzbacher, O., Nowak, M., \& Greene, J. (2014). Social Heuristics Shape Intuitive Cooperation. Nature Communications, 5, 3677. https://doi.org/10.1038/ncomms4677

Stagnaro, M. N., Arechar, A. A., \& Rand, D. G. (2017). From Good Institutions to Generous Citizens: Top-Down Incentives to Cooperate Promote Subsequent Prosociality But Not Norm Enforcement. Cognition, 167, 212-254.

https://doi.org/10.1016/j.cognition.2017.01.017 